Rev. Salud pública. 8 (2): 182-190, 2006

\title{
Discapacidad en población desplazada en el Cauca, Colombia
}

\author{
Leidy M. Obando-Ante ${ }^{1}$, Adriana E. Lasso-Benavides ${ }^{3}$ y Paola Vernaza-Pinzón ${ }^{4}$ \\ ${ }^{1}$ Fisioterapeuta. Clínica del Country, Bogotá, Colombia.E-mail: leidyobando82@yahoo.com \\ ${ }^{2}$ Fisioterapeuta. Hospital Universitario San José, Popayán, Colombia. \\ E-mail: adriannalasso@hotmail.com \\ ${ }^{3}$ Fisioterapeuta. Especialista en Epidemiología General. Departamento de Fisioterapia, Facultad de \\ Ciencias de la Salud, Universidad del Cauca, Popayán, Colombia. E-mail: pvernaza@unicauca.edu.co
}

Recibido 3 Marzo 2006/Enviado para Modificación 25 Mayo 2006/Aceptado 19 Junio 2006

\section{RESUMEN}

Objetivo Estimar la prevalencia de discapacidad en una población en situación de desplazamiento.

Métodos Se realizó un estudio descriptivo de prevalencia a 165 familias en situación de desplazamiento en la ciudad de Popayán, Cauca, Colombia entre junio y diciembre de 2003. La información se colectó a partir de una encuesta de variables sociodemográficas y biológicas.

Resultados La prevalencia de discapacidad en la población estudio fue del $8.5 \%$ respecto a los tipos de discapacidad, se encontraron prevalencias de $5.6,0,7$ y $1,2 \%$ para discapacidad física, sensorial y mental respectivamente.

Conclusiones La situación de discapacidad, sumada al desplazamiento es un problema de salud pública, pues además de afectar a un amplio grupo de individuos y familias, tiene un impacto negativo sobre la sociedad al afectar la productividad y el desarrollo de capital humano.

Palabras Clave: Violencia, discapacidad, salud pública, Colombia (fuente: DeCS, BIREME).

\section{ABSTRACT}

Disability in a displaced population in Cauca, Colombia

Objective Estimating the prevalence of disability in a displaced Colombian population.

Methods A descriptive prevalence study was carried out amongst 165 displaced families in the city of Popayán, Cauca, Colombia between June and 
December 2003. The information was collected via a questionnaire regarding socio-demographic and biological variables.

Results There was $8.5 \%$ disability in the population being studied. Regarding the type of disability $5.6 \%, 0.7 \%$ and $1.2 \%$ prevalence was found for physical, sensory and mental disability, respectively.

Conclusions Being disabled, added to that of being displaced, has become a public health problem as it affects a large group of individuals and families and also has a negative impact on society by affecting the productivity and development of human resources.

Key Words: Violence, disabled person, public health, Colombia (source: $\mathrm{MeSH}, \mathrm{NLM}$ ).

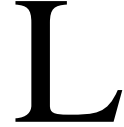

os cambios ocasionados en el perfil demográfico, especialmente los relacionados con las modificaciones de los patrones de mortalidad general y aumento de la esperanza de vida, traen consigo un incremento de la carga de discapacidad ocasionada fundamentalmente por la incidencia de lesiones afectivas, físicas y psíquicas producidas por la violencia, el conflicto armado y el desplazamiento, que reflejan una realidad social a la cual se le atribuyen múltiples causas pero que contribuyen a incrementar el riesgo de discapacidad.

En Colombia se realizó un estudio sobre el evento de discapacidad en nueve ciudades capitales donde se encontró que el 2,8 \% de la población estudiada presentaba algún tipo de discapacidad (1). Otro estudio realizado en el año 2001 en el departamento del Valle del Cauca arrojó una prevalencia de 3,9 \% (2). En el departamento del Cauca se realizo un estudio en el año 2003, encontrándose que la discapacidad mas frecuente fue la física con deficiencia en el sistema osteomuscular afectando las actividades de locomoción (3).

La población del departamento del Cauca, se encuentra expuesta al evento discapacidad dada la tendencia demográfica causada por el desplazamiento interno de los pobladores de las zonas rurales hacia la capital. De acuerdo a lo reportado por la Red de Solidaridad Social para el año de 2003, el municipio de Popayán (capital del departamento del Cauca) fue receptor de 11385 personas desplazadas, que incluyen 5591 hombres y 5794 mujeres, quienes conforman 2412 familias. Las familias desplazadas por la violencia se encuentran organizadas en 13 asociaciones distribuidas a lo largo del Municipio. (4). 
Entre las características de la población desplazada cabe destacar la presencia de niños y niñas, mujeres, ancianos y adicionalmente personas que sufren algún tipo de discapacidad ya sea congénita o adquirida probablemente a causa de la violencia.

Esta problemática justificó el desarrollo de la investigación con el objetivo de estimar la prevalencia de discapacidad en la población en situación de desplazamiento. Actualmente en el departamento del Cauca, no se han realizado estudios que determinen la prevalencia de discapacidad, ni en el ámbito general ni en la población en situación de desplazamiento.

\section{MÉTODOS}

Se realizó un estudio descriptivo de prevalencia en la población desplazada por la violencia del Municipio de Popayán en el periodo Junio-Diciembre de 2003. Se solicitó a la Red de Solidaridad Social Seccional Cauca (RSSSC), información sobre la población en situación de desplazamiento registrada a causa de la violencia y radicada en el municipio de Popayán. El universo estuvo constituido por las familias desplazadas a causa de la violencia residentes en Popayán. El total de familias desplazadas registradas en la RSSSC fue de 2412 de las cuales 548 fueron reportadas por la Red como desplazadas por la violencia según los criterios de la consultoría para los derechos Humanos y el desplazamiento (5). Las familias desplazadas por la violencia se encontraban organizadas en 13 asociaciones dispersas en asentamientos a lo largo del municipio. Debido a la magnitud de la población universo se efectuó un muestreo en etapas múltiples donde la unidad muestral la constituyo la familia. En la primera etapa se realizó un muestreo por conglomerados (13 asociaciones). Para el calculo de tamaño de muestra se tomo el $30 \%$ como valor esperado probable, se considero una confiabilidad del $95 \%$ y un máximo error admisible del $5 \%(6,7)$. El tamaño estimado de la muestra fue de 251 familias; la selección final se logró mediante el muestreo aleatorio estratificado. Fueron excluidas del estudio aquellos hogares en las que no se encontraba la persona cabeza de familia en el momento de la visita y familias que en el momento de la visita se retractaran del consentimiento para participar en el estudio. Finalmente 168 familias fueron reclutadas para el estudio correspondiente al $67 \%$ de la muestra. Para estimar la prevalencia de la discapacidad se estudiaron las siguientes variables: edad (de acuerdo al ciclo vital), sexo, presencia de discapacidad, tipo de discapacidad, signos síntomas padecidos comúnmente, número de integrantes de la familia, estado civil, ocupación previa al desplazamiento, ocupación actual, nivel de escolaridad, lugar de procedencia, hábitos nocivos y régimen de seguridad social en salud. Con las variables del estudio se diseño un instrumento utilizando 
preguntas ya validadas respecto a la caracterización familiar, detección de la discapacidad y condición de salud utilizada en el estudio de prevalencia del valle del Cauca. Para la aplicación del instrumento se realizaron reuniones previas en cada asociación donde se informo sobre los objetivos, métodos y resultados esperados del estudio. Una vez aprobado el consentimiento por cada una de las familias seleccionadas para participar en la investigación se procedió a programar visitas en los sitios de habitación para colectar la información.

La información obtenida fue suministrada por el jefe del hogar, aunque en ocasiones todos los miembros de la familia aportaban a la encuesta. En lo concerniente a la variable tipo de discapacidad se realizó una interpretación del lenguaje popular utilizado por la persona encuestada, extrayendo de esta la mayor información posible acerca de las dimensiones de la discapacidad. Por ejemplo, "las Piernas no le Funcionan" fue interpretado como dimensión física de la discapacidad, "hay que hablarle a gritos" dimensión sensorial de la discapacidad, "habla solo, grita y hace movimientos raros" como dimensión mental de la discapacidad, entre otros ejemplos.

La gran mayoría de las familias vivían en lugares de difícil acceso por encontrarse ubicados en asentamientos catalogados por el municipio como de alta peligrosidad. Además la desconfianza y miedo de las familias al tener que responder una encuesta hizo difícil completar la muestra estimada, lo que se convirtió en limitante para el estudio.

Todos los procedimientos de la investigación se realizaron de acuerdo con los principios bioéticos establecidos en la Declaración de Helsinki (8). Para el procesamiento y análisis estadístico de la información, se construyó una base de datos en el programa SPSS versión 11.0. Las variables se expresaron en frecuencias y proporciones. Posteriormente se estimo la prevalencia de discapacidad (9).

\section{RESULTADOS}

Respecto a la población en situación de desplazamiento, se encontró que las 168 familias incluidas en el estudio la conforman 756 personas, encontrándose que del total de la población encuestada 64 de ellas presentaron algún tipo de discapacidad. En la Tabla 1 se indican las familias en situación de desplazamiento.

Respecto a las características de los hogares encuestados se encontró una mayor proporción de familias extensas incompletas con un promedio de 5 
personas por hogar. En lo referente al sexo el 48 \% (363/756) pertenecían al sexo masculino y el 52 \% (393/756) al femenino. Se encontró que el 38,1 \% eran adultos (288/756), el 28,2 \% (213/756) niños, el 24,5 \% (185/756) adolescentes y un 9,3 \% (70/756) estuvo conformado por ancianos. El 68,3 $\%$ (516/756) de los encuestados tiene un nivel educativo de básica primaria, mientras que, solo el 1,3\% (10/753) ha realizado estudios técnicos.

Tabla 1. Familias en Situación de Desplazamiento organizadas en Asociaciones

\begin{tabular}{lcccccc}
\hline \multirow{2}{*}{ Asociación } & \multicolumn{2}{c}{ Familias } & \multicolumn{2}{c}{ Individuos } & \multicolumn{2}{c}{ Discapacitados } \\
\cline { 2 - 7 } & No. & $\%$ & No. & $\%$ & No. & $\%$ \\
\hline Andas & 3 & 1,8 & 24 & 3,2 & 1 & 1,5 \\
Asodesca & 22 & 13,1 & 80 & 10,6 & 18 & 28,1 \\
Caminos de Esperanza & 10 & 6,0 & 45 & 6,0 & 2 & 3,1 \\
Fe y Esperanza & 11 & 6,5 & 37 & 4,9 & 1 & 1,5 \\
Luz de Esperanza & 6 & 3,6 & 34 & 4,5 & 2 & 3,1 \\
Nueva Floralia & 20 & 11,9 & 68 & 9,0 & 1 & 1,5 \\
Nueva Jerusalén & 2 & 1,2 & 12 & 1,6 & 3 & 4,7 \\
Nueva Vida & 18 & 10,7 & 73 & 9,7 & 0 & 0 \\
Nuevo Porvenir & 11 & 6,5 & 59 & 7,8 & 6 & 10,1 \\
Nuevo Putumayo & 40 & 23,8 & 186 & 24,6 & 11 & 17,1 \\
Semillas de vida & 11 & 6,5 & 67 & 8,9 & 15 & 23,4 \\
Vida y Paz & 11 & 6,5 & 61 & 8,1 & 2 & 3,1 \\
Villa España & 3 & 1,8 & 10 & 1,3 & 2 & 3,1 \\
Total & 168 & 100 & 756 & 100 & 64 & 100 \\
\hline
\end{tabular}

Fue curioso encontrar que solo el 3,6 \% (27/756) de la población refirió tener hábitos nocivos como el consumo de alcohol, tabaco y/o sustancias psicoactivas. El 70,1 \% (530/756) de la población refirió problemas en su estado de salud, los síntomas relatados en orden de frecuencia fueron: tos, dolor de cabeza, dolor de estomago, diarrea y dolor articular (datos no mostrados).

En la Tabla 2 se describe la prevalencia de discapacidad, del total de la población encuestada el 8,5 \% presento algún tipo de discapacidad (IC 95 \%: 6,6-10,4). En lo que se refiere al sexo, no se encontraron diferencias significativas, la discapacidad se encontró con mayor frecuencia en los ancianos, probablemente por los cambios degenerativos que se dan a este nivel del ciclo vital. Respecto al tipo de discapacidad el estudio encontró que la población en situación de desplazamiento se ve más afectada por discapacidades de tipo físico que mentales o sensoriales.

En cuanto a las características de la población con discapacidades el 64,1 \% (41/64) tiene un nivel de escolaridad básico, mientras que el 29,7 \% $(19 / 64)$ no ha realizado ningún tipo de estudio. El perfil ocupacional de la población después del desplazamiento tuvo serios cambios en su actividad ocupacional el 37,5 \% (24/64) eran agricultores y después del desplaza- 
miento sumado al evento discapacidad el 20,8 \% (5/24) se encuentran desempleados, el 45,8 \% (11/24) son vendedores ambulantes y el 33,4 \% realizan oficios varios. En lo referente a los hábitos nocivos el 82,8 \% (53/64) de los discapacitados consume alcohol, solo el 15,6 \% (10/64) pertenece al régimen subsidiado de salud y en caso de enfermedad el 25,0 \% (16/64) asiste al puesto de Salud, el 20,3 \% (13/64) acude al curandero, el 18,7 \% (12/64) se automedica, el 14,1 \% (9/64) asisten donde un farmaceuta y el 12,5 \% (8/64) asiste al hospital. Los síntomas presentes en la población discapacitada según orden de importancia fueron: Dolor articular, tos, y dolor de espalda (datos no mostrados).

Tabla 2. Prevalencia de discapacidad en la población desplazada por la violencia

\begin{tabular}{|c|c|c|c|}
\hline \multirow{2}{*}{ Características } & \multirow{2}{*}{$\mathrm{n}$} & \multicolumn{2}{|c|}{ Prevalencia } \\
\hline & & $\%$ & IC $95 \%$ \\
\hline \multicolumn{4}{|l|}{ Discapacidad } \\
\hline Presencia & 64 & 8,5 & $6.6-10.4$ \\
\hline \multicolumn{4}{|l|}{ Sexo } \\
\hline Masculino & 32 & 8,1 & $5,4-10,8$ \\
\hline Femenino & 32 & 8,8 & $5,9-11,7$ \\
\hline \multicolumn{4}{|l|}{ Grupo etáreo } \\
\hline Niños & 2 & 0,9 & $0,4-2,2$ \\
\hline Adolescentes & 7 & 3,8 & $1,1-6,5$ \\
\hline Adultos & 28 & 9,7 & $6,3-13$ \\
\hline Ancianos & 27 & 38,5 & $27-49$ \\
\hline \multicolumn{4}{|c|}{ Tipo de discapacidad } \\
\hline Física & 50 & 5,6 & $4,0-7,2$ \\
\hline Sensorial & 5 & 0,7 & $0,1-1,3$ \\
\hline Mental & 9 & 1,2 & $0,4-1,9$ \\
\hline
\end{tabular}

\section{DISCUSION}

La discapacidad es un problema que afecta a gran parte de la población mundial y su magnitud se acrecienta por sus repercusiones en la familia y en la sociedad, incrementando los costos de atención médica sumada a una sobrecarga económica y social (10).

Los resultados del presente estudio muestran las características generales de la población desplazada conformada en su mayoría por mujeres, adultos, con niveles educativos básicos y con problemas en su estado de salud. Las poblaciones vulnerables con familias extensas, bajos niveles de escolaridad y desempleo aumentan el riesgo de pobreza y están asociados a la condición de discapacidad $(11,12)$.

La prevalencia encontrada en nuestro estudio supera a la encontrada en el estudio demográfico: "Sistema de Información de Discapacidad“(13). Pero 
se acerca a la encontrada en el estudio de "Prevalencia de Discapacidad en el departamento del Valle del Cauca”, lo cual puede indicar que la discapacidad es un problema social que afecta a los estratos más bajos y no esta directamente asociada al desplazamiento a causa de la violencia. Esto puede explicarse en el diseño del instrumento, en el que se incluyeron preguntas ya validadas y utilizadas en el estudio del valle del Cauca, pero en la cual se omitieron aspectos tales como: Deficiencia y Discapacidad previa al desplazamiento y después del desplazamiento y causas que condujeran al evento discapacidad.

Cabe mencionar que nuestro estudio arrojo una prevalencia de $38,5 \%$ en la población anciana probablemente por las deficiencias en los sistemas orgánicos propias del envejecimiento (14), que conllevan a alteraciones en las estructuras del cuerpo y redundan en limitaciones de la actividad restringiendo la participación social.

Dentro de las nuevas perspectivas taxonómicas y terminológicas de la Clasificación Internacional del Funcionamiento y los estados de Salud (15) se encuentra el "Bienestar" que engloba el universo de los dominios de la vida humana incluyendo aspectos físicos, mentales, sensoriales y sociales que componen lo que se puede llamar una "buena vida". En nuestro estudio se encontró que la discapacidad mas frecuente fue la física (limitación para el cuidado personal, para la locomoción, para la disposición del cuerpo y para la destreza), seguida de la mental (trastornos en el comportamiento adaptativo) y sensorial (visual y/o auditiva), diferente a lo reportado por Cardozo y Colaboradores (16) quienes encontraron mayor presencia de discapacidad mental luego del conflicto bélico. Rivera y Camargo (17) en el estudio de caracterización de la Discapacidad en poblaciones de estratos 1 y 2, encontraron que la discapacidad más frecuente fue la sensorial seguida de la física. Mientras que en el estudio del departamento del Valle, la discapacidad mas frecuente fue la física relacionada con las estructuras y funciones neuromusculoesqueléticas. Respecto a los dominios sociales del bienestar se encontró que la población se encuentra organizada en asociaciones, lo que les permite interactuar de acuerdo a las normas sociales, pero es preocupante como el desplazamiento obliga a la población con algún tipo de discapacidad al abandono de las actividades laborales típicas de las zonas rurales, teniendo que adaptarse a sus nuevas condiciones socio-culturales, sumado a la búsqueda de empleo para poder satisfacer los mínimos básicos de supervivencia.

Colombia actualmente enfrenta grandes problemas sociales, pero tal vez las instituciones democráticas no están al tanto de la situación de discapaci- 
dad en la población desplazada. Si la visión 2019 (18) es cerrar las brechas sociales, el estado debe comprometer a la sociedad a ser solidaria con las poblaciones vulnerables. Esto implica la consolidación de un sistema de información veraz en tiempo real, para que se protejan los derechos de la población discapacitada

La población desplazada por la violencia, incluye en su núcleo social niños, adolescentes, adultos y ancianos población que carece de oportunidad para acceder a los servicios de salud, educación y proyectos de generación de ingresos aumentando el riesgo de padecer discapacidad lo cual se constituye en un problema de salud pública, pues además de afectar a un amplio grupo de individuos y familias, tiene un impacto negativo sobre la sociedad al afectar la productividad y el desarrollo de capital humano. Lo que justifica que las instituciones locales, departamentales, regionales y nacionales desarrollen programas de vigilancia y control del evento discapacidad para analizar el impacto social y económico que este produce. Se recomienda para próximas investigaciones, contar con un equipo multidisciplinario que complemente la encuesta sobre el evento discapacidad con una evaluación de las deficiencias por sistemas, estructuras y funciones, limitación de la actividad, restricción de la actividad y factores contextuales, que permitan establecer el compromiso de la discapacidad en poblaciones vulnerables •

Agradecimientos. Los autores desean expresar su gratitud a Red de Solidaridad Social seccional Cauca, las personas en situación de desplazamiento que participaron en el estudio, al Profesor Fernando Pinzón y al Doctor Carlos Hernán Sierra.

\section{REFERENCIAS}

1. Ministerio de Educación Nacional, Universidad Javeriana. Sistema de información de discapacidad. Corporación Interinstitucional de Rehabilitación Bogotá DC; 1994.

2. Universidad del Valle, Secretaria Departamental de Salud del Valle. Prevalencia de Discapacidad en el departamento del valle del Cauca. Cali; 2001

3. Vernaza P, Paz C. Funcionamiento y Discapacidad en pacientes valorados por Fisioterapia. Revista de la Facultad de Ciencias de la Salud Universidad del Cauca. 2004 5(1): 8-17.

4. Red de solidaridad Social Seccional Cauca. Estadísticas Población en situación de desplazamiento. Popayán, 2003.

5. Consultoría para los Derechos Humanos y el Desplazamiento CODHES. La tendencia nacional del desplazamiento interno. Bogota DC, 2001

6. Milton JS. Estadística para Biología y Ciencias de la Salud. Madrid: Mc Graw Hill; 2001. p 267-269. 
7. R. Polit D, Hungler P. Investigación científica en ciencias de la salud. México: Mc Graw Hill; 2000. p 274-280.

8. The Helsinki Declaration. Orvosi hetilap. 1965;106(36):1715-1716

9. Tapia Granados JA. Medidas de prevalencia y relación incidencia-prevalencia. Med Clin (Barc) 1995; 105: 216-218.

10. Organización de Naciones Unidas. Normas uniformes sobre la igualdad de oportunidades para las personas con discapacidad resolución 48/96. New York, 1996.

11. Velez C. Colombia poverty. Report volume I World Bank. ;2002. p. 44.

12. Hernández J, Hernández I. Una aproximación a los costos indirectos de la discapacidad en Colombia. Rev. Salud Pública. 2005; 7(2):130-44.

13. Ministerio de Salud. Ocupación, discapacidad y costos. Bogota DC; 2000.

14. Vasallo C, Sellanes M. La salud en la tercera edad. Argentina: Secretaria de la tercera edad; 2001. p 2-127

15. Organización Mundial de la Salud. Clasificación Internacional del Funcionamiento y la Discapacidad. Ginebra, 2000

16. Cardozo BL, Bilukha OO, Crawford CA, Shaikh I, Wolfe MI, Gerber ML, Anderson M. Mental health, social functioning, and disability in postwar Afghanistan. JAMA. 2004 Aug 4; 292(5):626-8.

17. Rivera J, Camargo D. Caracterización de la discapacidad en la población sisbenizada niveles I y II. Bucaramanga; 2001.

18. Dirección Nacional de Planeación, Presidencia de la Republica. Visión Colombia II centenario Plan 2019. Bogotá, DC; 2005. 